\title{
Faire de votre système d'information un levier de performance dans la réalisation de votre projet de dématérialisation
}

\author{
Mots-clés \\ gestion publique - gestion locale - dématérialisation - financement participatif \\ Le système d'information peut être un levier de performance dans le cadre \\ d'un processus de numérisation, que ce soit au sein de l'administration \\ d'un département ou dans des villes qui font appel à une plateforme de \\ financement participatif.
}

Ta eudi $1^{\text {er }}$ juin 2017, s'est tenue la rencontre intitulée "Comment faire de votre système d'information un levier de performance dans la réalisation de votre projet de dématérialisation?" organisée dans le cadre des Rendezvous de la Transformation de la Fonction Finance.

\section{Nicolas LAROCHE}

Délégué Général du FORUM pour la Gestion des Villes et des Collectivités Territoriales

Nicolas LAROCHE a ouvert les échanges en rappelant que, dans le cadre des Rendez-vous de la Transformation de la Fonction Finance, la dématérialisation de la Fonction Finance a d'abord été traitée d'un point de vue comptable (RDV de mars 2017), puis d'un point de vue ressources humaines/ accompagnement des équipes (RDV d'avril 2017) et maintenant d'un point de vue système d'information.
En effet, un système d'information performant constitue un puissant levier dans la mise en place de processus de dématérialisation. II permet à la fois une automatisation des tâches, une sécurisation des process et la protection des données.

Pour autant, les démarches de dématérialisation ne peuvent être simplement réduites aux systèmes d'information. Une démarche de dématérialisation réussie engage en effet l'ensemble des services d'une organisation.

Dès lors, comment faire d'un système d'information un levier de performance de l'action de la collectivité ? Quels sont les axes d'un schéma directeur à l'ère du numérique ? Quelle est la place du service informatique dans ces projets transversaux? Pour répondre à ces interrogations, deux grands témoins sont venus apporter leur expérience. 


\section{Bertrand SOPEL}

Directeur Adjoint de l'innovation numérique et des systèmes d'information au Conseil Départemental de Seine-Saint-Denis

Bertrand SOPEL est venu détailler la stratégie numérique déployée sur le territoire, ainsi que la place et le rôle de sa direction dans le projet de dématérialisation de la fonction finance.

1. Il y a 7 ans, les systèmes d'information étaient, au sein du Conseil Départemental, un simple service rattaché au pôle ressources et moyens. Deux ans plus tard, ceux-ci deviennent la « Direction des systèmes d'information ». La direction passe alors de 80 ETP $^{1}$, à plus de 130. Depuis 2 ans, les choses s'accélèrent : la direction a été rattachée au pôle stratégie et innovation, puis s'est transformée en "Direction de l'innovation numérique et des systèmes d'information ».

Cette direction englobe alors l'aménagement numérique, l'innovation au sens large et permet aux agents d'y intégrer un « service public lab » dont la fonction principale est de permettre l'expérimentation sur le territoire, tout en incluant un pôle dédié à la stratégie numérique.

2. La stratégie numérique du département, votée par l'assemblée départementale, repose sur quatre grands axes:

- fédérer les acteurs du numérique sur le territoire (écoles, entreprises, data centers, start-ups, associations dédiées à l'innovation digitale, etc.) et créer des synergies entre eux. Un Conseil du Numérique (ou do tank) a par exemple été créé en 2016.

- mettre les biens communs numériques à disposition de tous. Il est mis par exemple en place des conventions et accords avec les écoles du numérique, afin de mêler apprentissage et prototypage pour créer des services aux usagers.

- faciliter la diffusion de la culture numérique;

- impulser les services numériques du $21^{\mathrm{e}}$ siècle 2 .

La finalité est de créer un environnement commun et permettre à tous les publics d'œuvrer ensemble.

3. Les impulsions se font projet par projet. Parmi les projets aujourd'hui lancés, on trouve des formations au numérique, au service de la réinsertion. Ces formations sont le fruit d'une collaboration entre start-ups et associations pour l'intermédiation. Elles visent à accompagner les usagers dans la prise en main du numérique. Certains agents de la collectivité bénéficient également de ces formations.

Autre projet à destination des usagers, notamment des collégiens de classe de $3^{e}$, la création d'une plateforme dédiée à la recherche du stage obligatoire en cours d'année. Elle permet aux collégiens de postuler au sein des entreprises présentes sur le territoire, comme la SNCF, Arcelor Mittal, Siemens, Ventes Privées ou SPIE....

Le Département a également mis en place une plateforme permettant aux usagers de dématérialiser les échanges avec l'administration, pour trois services en particulier : la carte Améthyste, un guichet unique et dématérialisé pour la demande de subventions et un autre concernant les demandes liées au handicap.

4. En interne aussi des process et des métiers ont évolué pour se dématérialiser. Au 1er janvier 2017, conformément au calendrier fixé par l'État, la dématérialisation a concerné la fonction comptable, dont les processus métiers ont été entièrement digitalisés.

Les protocoles en matière de formats et de sécurisation sont drastiques, surtout concernant la comptabilité. Lors de la dématérialisation des processus, certaines problématiques sont apparues, notamment concernant la mise en place de la signature électronique.

La dématérialisation complète au sein du Conseil Départemental, bien que complexe à mettre en place, a été cependant perçue de manière générale comme un projet positif en termes de maîtrise des coûts et d'évolution des métiers.

C'est au fil des dialogues entre services et agents qu'ils se sont aperçus du levier que cette occasion représentait. Le processus métier pouvait être réorganisé, tout comme l'organisation comptable.

L'objectif a donc été, à travers la dématérialisation, de réduire les risques et erreurs, les oublis, les pertes de documents et enfin, faciliter la relation à l'usager. C'est pourquoi, dès le début, le Conseil Département a choisi d'automatiser rapidement les tâches afin d'optimiser les coûts.

Cette transformation des métiers a nécessité un accompagnement des agents comptables (environ 350). Leurs missions sont désormais davantage tournées vers le contrôle et l'audit.

Parmi les autres grands chantiers à mettre en place figurent la gestion électronique des documents (nommage, classement, pérennité du stockage électronique, problématique liée à la sécurisation des données, etc.).

5. La gouvernance du projet n'a pas été simple au début. II a donc a fallu définir et structurer une vraie gouvernance, soutenue par la Direction générale des Services.
1 Équivalent temps plein

2 Retrouvez la présentation de la stratégie numérique du département:

https://pandasuite.com/viewer 10.8.46/58b841c1aacf36cf0b00 $0459 ? \mathrm{t}=1$ 
"Le soutien de la Direction Générale des Services est indispensable » Bertrand SOPEL

La place des élus est également essentielle, puisque ce sont eux qui ont été les moteurs de ces projets. En effet, ils ont été un puissant levier pour, entre autres, rassurer l'écosystème privé dans le département, concernant notamment les questions de délais de paiement, surtout les PME, très nombreuses sur le territoire.

Il a également fallu nommer un pilote ayant la responsabilité complète sur la gouvernance du projet, tant sur le plan technique que fonctionnel. Désigné au sein de la Direction de l'Innovation, celui-ci a dû veiller à coordonner l'ensemble des projets, tout en restant à l'écoute afin d'assister celles et ceux qui en avaient besoin.

Ce pilote a porté, avec les équipes et les services, le projet de dématérialisation, tout en définissant un plan d'accompagnement et de formation, tout en souplesse, auprès de plus de 500 utilisateurs et 350 agents comptables.

Ce plan s'est concrétisé sous la forme de café tuto le matin, de tutoriels accessibles en ligne, de plusieurs piqûres de rappel et de coaching. Enfin, surtout, il a fallu créer un vocabulaire commun pour tous.

"Le projet se traduit par 30 à $40 \%$ de méthode agile et 60 à $70 \%$ de formations et d'accompagnement » Bertrand SOPEL

6. Plusieurs indicateurs ont été mis en place afin de mesurer la performance du système d'information. Dès le début, la question s'est posée, à la fois dans l'outil d'entrée des factures, Kofax, qui permet d'accéder à des indicateurs et statistiques (quelle indexation a été donnée ? Quelles sont les erreurs relevées ? etc.) et dans Alfresco dont les indicateurs permettent de relever diverses informations concernant les stocks, les blocages dans les services, ou encore, les chaînes de paiement.

7. Concernant la sécurisation des données, le Conseil Départemental a fait le choix de rendre public ses données, après validation auprès d'un comité éditorial qui vérifie ce qui peut ou ne peut pas être publié. La collectivité a également créé une cellule de crise, pour gérer les attaques et la sécurité de l'ensemble des systèmes. Cependant, tout comme la mise en place de processus et la réorganisation des services, une politique sécuritaire se crée petit à petit.

\section{Julien QUISTREBERT \\ Président-Fondateur de Collecticity}

Julien QUISTREBERT est venu expliquer comment une plateforme numérique comme Collecticity contribue à la performance de l'action publique et à la dématérialisation des collectivités territoriales.

1. Plateforme de crowdfunding lancée en septembre 2016, Collecticity permet aux collectivités territoriales d'obtenir des prêts citoyens pour des projets variés, qui ont souvent comme particularité d'être des projets difficiles à financer par des systèmes traditionnels. II s'agit de financement par les citoyens qui souhaitent s'impliquer dans la vie de la cité. Cependant, il est également possible de faire une demande de prêt, lorsqu'un appel aux dons semble complexe.

En tant que représentantes de l'intérêt général, Collecticity offre aux collectivités une solution intermédiaire et la possibilité, aux citoyens, de faire de l'investissement sans risque, tout en donnant un sens à celui-ci.

2. En faisant appel aux citoyens et en usant des possibilités qu'offrent Collecticity, les collectivités participent à la démarche de Smart City. Aujourd'hui, les collectivités sont dans un système vertical, alors même que le concept de Smart City s'y oppose en prônant un système horizontal.

La smart city ne doit pas se réduire au partage d'informations en temps réel et l'open data. La vraie smart city doit être axée principalement sur la communication et se servir de la technologie pour remettre le citoyen au cœur de ses préoccupations.

Le système participatif permet donc de réintégrer les citoyens au cœur de la vie de la cité tout en les fédérant autour d'un projet et chacun peut mieux comprendre la façon dont les impôts locaux sont utilisés. En effet, en faisant appel au financement participatif, les collectivités font preuve de transparence et tissent les liens d'une démocratie participative durable.

3. Concrètement, lorsqu'une collectivité souhaite lancer un appel aux dons ou contracter un prêt citoyen, son projet est soumis à validation auprès des équipes de Collecticity qui en étudie la solvabilité et la durabilité. Dès que celui-ci est validé, la mise en ligne se fait de façon dématérialisée. Chaque pièce justificative des dossiers est sous format dématérialisé et transite via la plateforme. Ce genre de process, qui est assez novateur, n'en demeure pas moins plus complexe qu'il n'y paraît.

"La vraie pédagogie n'est pas d'expliquer aux collectivités comment lancer un appel aux dons sur la plateforme, mais plutôt "pourquoi le faire?" " Julien QUISTREBERT

4. La première collectivité à avoir « osé » franchir le pas est Saint Brieuc Agglomération qui souhaitait 
financer des panneaux solaires. Le projet avait un sens, prouvait que la collectivité s'investissait dans le renouvelable et était facile à expliquer aux usagers.

La commune de Langouet, en Bretagne, qui compte environ 600 habitants et est aguerrie aux questions d'écologie, a également fait un appel aux dons pour lever $40000 €$ afin de créér un habitat, au bénéfice de l'environnement, dans une démarche Cradle To Cradle ("Du berceau au berceau") qui consiste à placer au cœur de toutes les étapes de vie d'un produit (conception, production et réutilisation) une logique écologique avec $0 \%$ de pollution et $100 \%$ de réutilisation.

Les projets étant novateurs, répondant aux demandes des citoyens et surtout, reposant sur un système de donnant-donnant (le citoyen comprend quels sont les bénéfices de ces prêts et la collectivité évite de devoir emprunter auprès d'une banque au risque de contracter des emprunts longs et coûteux), ceux-ci arrivent à se concrétiser

"Les collectivités territoriales doivent apprendre à diversifier leurs sources de financement »

\section{Julien QUISTREBERT}

Si le financement participatif - don ou emprunt est une solution très intéressante pour les petites villes ou communes rurales, les grandes villes peuvent également y trouver leur intérêt. En effet, ce genre de plateforme dématérialisée leur permet de créer un vrai échange avec les habitants, les incitant à s'impliquer davantage dans la vie de la cité.

5. Après plus d'un an et demi de développement pour créer la plateforme (temps moyen constaté notamment pour les échanges avec les ministères concernant les questions juridiques de mise en place) Collecticity est aujourd'hui en phase "d'évangélisation » auprès des élus. Ce sont en effet eux qui impulsent auprès des services les lancements de projets de ce type.

Alors que les grandes villes ont des prises de décision longues, ce sont les petites communes qui font plus facilement confiance à Collecticity et n'hésitent pas à solliciter la plateforme. Ce sont souvent elles qui ont un besoin de financement plus critique et une capacité de prise de décision plus rapide.

Quant aux prêteurs traditionnels, les réactions sont variables. Certains voient en Collecticity un concurrent sérieux, d'autres s'en amusent ou commencent à réfléchir à l'idée de lancer un concept similaire. Cependant, Julien Quistrebert rappelle volontiers que sa plateforme ne sera pas une « licorne » (terme créé en 2013 par Aileen Lee, spécialiste américaine du capital-risque, afin de qualifier les startups valorisées à plus d'un milliard de dollars) à revendre et qu'il cherche plutôt volontiers des partenaires avec qui il pourra faire avancer le concept.

L'intégralité des débats est accessible en ligne, sur demande, dans la chaîne : «Les Rendezvous de la Transformation de la Fonction Finance »

\section{Contact : nicolas.laroche@forum-gv.com}

Le compte-rendu de la première rencontre «Dématérialisation et digitalisation de la fonction finance : enjeux et opportunités pour le bloc local » a été publié dans le n³-2017 de Gestion \& Finances Publiques, p. 41. 